IFT-P.050/97

IFUSP/P-1279

\title{
Are High Energy Cosmic Rays Magnetic Monopoles?
}

\author{
C.O. Escobar ${ }^{1,2}$ and R.A. Vázquez ${ }^{1,3}$ \\ ${ }^{1}$ Instituto de Física, Universidade de São Paulo, \\ Caixa Postal 66318 \\ 05315-970 - São Paulo, S. P. \\ Brazil \\ 2 Instituto de Física "Gleb Gataghim' \\ Universidade Estadual de Campinas, Unicamp \\ 13083-970 - Campinas, S. P. \\ Brazil \\ ${ }^{3}$ Instituto de Física Teórica, \\ Universidade Estadual Paulista \\ Rua Pamplona, 145 \\ 01405-900, São Paulo, S.P. \\ Brazil
}

\begin{abstract}
We argue that magnetic monopoles can not be associated to the highest energy cosmic rays as recently suggested. Both the observed spectrum and the arrival direction disagree with observation.
\end{abstract}

\section{INTRODUCTION}

The highest energy cosmic rays are subject to several speculations about their origin and acceleration mechanism. Perhaps the most studied models, besides conventional models, are 
the top-down models (TD), where the particles are generated at extremely large energy and loose part of it by interacting with the medium. A central characteristic of these models is the (observed) flat spectrum they predict at the largest energies. The way TD models obtain the power to accelerate particles depends on the model but in all of them it is related to some topological defect produced with gauge symmetry breaking of a Grand Unification Theory in the early stages of the Universe.

Recently, it was suggested that these cosmic rays could be due to relativistic magnetic monopoles interacting with the air [1.21. Here it is assumed that it is the monopole which directly will produce the shower by interacting with air in some not completely specified way. The monopoles could originate a shower by some catalysis mechanism or initiate a electromagnetic shower. It is assumed that showers induced by monopoles have the same characteristics as regular showers. The monopoles easily pick up energy in the galactic magnetic fields due to the large charge $\left(\alpha_{M} \sim 1 / \alpha\right)$ of the monopole. With a relatively low monopole mass $\mathrm{M} \sim 10^{10} \mathrm{GeV}$ typical galactic magnetic fields will accelerate the monopole to $\gamma \sim 10$ in a $300 \mathrm{pc}$ distance, easily acquiring enough energy to produce the observed events. The proposed mechanism is based on three "coincidences" [2] i) In $300 \mathrm{pc}$ the monopoles will acquire enough energy to produce the showers, as said above. ii) The observed HECR flux is of the same order of magnitude as the flux allowed by the Parker limit [3, [4] on monopoles for low mass monopoles, $\mathrm{M} \sim 10^{10} \mathrm{GeV}$. iii) The limit on the mass of the monopole given by the Kibble mechanism [5] for their generation in the early Universe gives the same order of magnitude as the previous points, so that monopoles do not overclose the Universe.

This interesting suggestion could avoid problems with the GZK cut off which are present for other scenarios and with the maximum reachable energy. We argue, however, against this idea. We will see below that monopoles will have a different spectrum, much flatter, and moreover a visible anisotropy in the direction of the magnetic fields. 


\section{SIMULATION}

We have developed a Monte Carlo program to propagate monopoles in the galactic medium. The galactic magnetic field is parametrized using the models given by Stanev [6] and Vallée [7]. For our calculations we will used both models given in ref. [6], the ASS_S and BSS_A models. The ASS_S model (model A, from here on) is axisymmetric and symmetric in the $z$ component whereas the BSS_A model (model B) is bisymmetric and has odd parity

in the $z$ component. In our simulations we will neglect the $z$ component. A random magnetic field is added to simulate the random component. The random field is chosen with a random direction in a lattice of $300 \mathrm{pc}$ and a magnitude chosen to be of the order of $20 \%$ of the regular component. Monopoles are followed in the magnetic field by numerically solving the equations of motion using an adaptive Runge Kutta method. The step is forced to be always much lower than the size of the random component lattice, $300 \mathrm{pc}$, in order not to overstep the random lattice.

\section{A. Scenario A}

Let's assume first that near the center of the galaxy there is an outgoing isotropic flux of monopoles. This would be the case if, for instance, a supernova exploded liberating its (possibly bounded) monopoles, as pointed in ref. [1]. Monopoles are then accelerated and followed until they reach a distance equal to the distance to the Earth from the center of the galaxy. We found that for both magnetic fields the spectrum of the monopoles reaching Earth is very different from the observed spectrum. In fig.1 we can see the spectrum of the hypothetical monopoles for magnetic fields model A (figures for the model B are similar). For the sake of comparison we also show a typical cosmic ray flux spectrum (with slope -2.7 ) and normalized to the same number of events. We see that for monopoles the spectrum peaks at a very high energy, indeed much higher than the maximum observed energy. In order to see 8 events with energy higher than $100 \mathrm{EeV}$ we will have to see many more of 
energy higher (even $1000 \mathrm{EeV}$ ). We should point out that this result is independent of the mass of the monopole, since the kinetic energy acquired by the monopole does not depend on the mass. We can see that monopoles accelerate at much higher energies than those quoted by [1,2] and with an spectrum which is essentially flat over a large energy region $(\sim$ one order of magnitude). This spectrum is clearly not compatible with the observed spectrum, which falls off steeply with energy (slope $\sim-2.7$ ). The result is independent of the assumed magnetic field model and it is true so long as the random component is smaller than the regular component.

The arrival direction of monopoles in this scenario is plotted in fig.2 where we plot the $\phi$ angle distribution $\left(\phi=0^{\circ}\right.$ corresponds to the galactic center $)$ for the model $\mathrm{A}$. We can see that there are "forbidden" directions: monopoles don't come directly from the galactic center. Also they don't come at $\phi=180^{\circ}$. For both models the galactic magnetic field at the position of the Earth is directed almost in the $\phi=90^{\circ}$ direction (with a small pitch angle) which aligns the monopoles irrespectively of their initial direction.

One may argue that there is no reason why monopoles should be distributed near the center of the galaxy. Due to their low mass, these monopoles are always relativistic and therefore they are not confined by the gravitational galactic field which is in fact negligible for them. And probably they are not confined either in supernovas. We can see this easily. A monopole is accelerated by a magnetic field to:

$$
\gamma-1 \sim \frac{q_{M} B L}{M} \sim 0.02 \frac{B}{310^{-6} G} \frac{L}{1 p c} .
$$

In other words, in only 1 pc the monopole could accelerate to a velocity much above the escape velocity for typical galactic magnetic fields. This also justifies a posteriori our neglecting of the gravitational field.

\section{B. Scenario B}

Let's assume next that monopoles are accelerated somehow in the extragalactic magnetic field and that they penetrate through the galaxy and arrive to Earth. We simulate this by 
back propagating the monopoles from the Earth with an energy $E_{0}$ back in time until a given distance is reached. We find that for almost all arrival directions the monopoles, in fact, have to loose energy in order to reach the Earth. This is a consequence of the "spiral" form of the magnetic field: Only a small cone of angles are in favour of the magnetic field, and this is only for a (relatively) small distance, for the magnetic field changes direction and then the monopole finds itself running "against the wind". This is independent of the sign of the magnetic charge of the monopole and also of the model chosen for the magnetic field.

Then for most of the arrival directions we find that monopoles are in fact deaccelerated in the galactic magnetic field and that in order to arrive at Earth with, say, $\sim 100 \mathrm{EeV}$ they needed to have much bigger energies initially. This can be seen in fig. : 3 where we plot the energy distribution for monopoles arriving at Earth with energy $100 \mathrm{EeV}$ and back propagated to a distance of $300 \mathrm{pc}(\mathrm{a}), 900 \mathrm{pc}(\mathrm{b})$ and $3 \mathrm{kpc} \mathrm{(c).} \mathrm{At} \mathrm{a} \mathrm{distance} \mathrm{of} 3 \mathrm{kpc}$ only a small fraction are really accelerated (the average energy to reach the Earth with 100 $\mathrm{EeV}$ is $270 \mathrm{EeV})$.

\section{COMPARISON WITH OBSERVED HECR}

What about the observed high energy events? There are 8 observed events above 100 $\mathrm{EeV}$. All, but one, arrive in a direction almost perpendicular to the local magnetic field which implies that irrespectively of being a monopole or an antimonopole these directions are not favoured by the local magnetic field. Only the Akeno event (pair event n.3 in ref. [8]) is in a direction favoured by the local magnetic field, if this event was an antimonopole. This must be necessarily an odd and very improbable situation because one should expect monopoles to come aligned with the local magnetic field. In fact observation suggest that the arrival direction of the highest energy events are correlated with the supergalactic plane [9] which for the observed region of the supergalactic plane is almost perpendicular to the local magnetic field.

Events with energy lower than around $100 \mathrm{EeV}$ are not assumed to be monopoles since 
they would not have energy enough to initiate showers. But the Stanev et al. [9] study (and see also the Akeno study of their events [8]) suggest that the correlation is valid for lower energy events too $(E>40 \mathrm{EeV})$, hinting towards the same origin for both types of events. In addition many of the highest energy events are paired with other of lower energy. This is true for the Fly's Eye event (which is paired with one Akeno event and with the Yakutsk event) and for the second's world highest energy event. These correlations can be easily accounted for in a conventional scenario whereas in the monopole case they are difficult to explain.

Let's consider the Fly's Eye event. It has the same arrival direction (within errors) as the highest event detected by Yakutsk, but it has a higher energy. It is obvious that if we back propagate them from the Earth to a given distance, the original position for both events must be very different (and also the original momentum). So we have the strange situation of two monopoles coming from very different points of the galaxy but they manage to arrive at the same point with the same direction. As mentioned before, this direction is not favoured by the magnetic field. We can see in fig. 4 the trajectory for both events in the $x y$ plane and in fig.5 the energy as a function of the distance to the Earth (for both a monopole and an antimonopole origin). In any case, the particle must be deaccelerated by the magnetic field. Certainly it must be very improbable such situation but giving an actual number for the probability is difficult.

In order to give a partial answer, we ran the following simulation. Monopoles are seeded at a random position in a cube of $20 \mathrm{kpc}$ centered in the Galaxy with an initial random energy which is assumed to be a gaussian of mean energy $100 \mathrm{EeV}$ (this is justified by the previous simulations). They are propagated in the galactic field until i) the distance to the Earth is bigger than $20 \mathrm{kpc}$ where they are assumed to go out of the galaxy and are dropped or ii) the distance to the Earth is less than $4 \mathrm{kpc}$ and then they are kept. The flux at the Earth is assumed to be same as the flux in the sphere of $4 \mathrm{kpc}$ around the Earth. The final distance, $4 \mathrm{kpc}$, is a compromise between computer time and getting the closest possible to the Earth, but it is a conservative assumption. Getting closer to the Earth can only collimate 
more the fluxes and therefore give more acute differences with the observed flux. We present in fig.6 the arrival direction in galactic coordinates of the monopoles which satisfy the above criteria for the model A. For the sake of comparison we also show the arrival direction of the most energetic cosmic rays which were available to us. As said previously we observe that no monopole arrived in the direction of the observed events, all of them arrive with either $\sim 90^{\circ}$ or $\sim 270^{\circ}$ longitude, as expected, mainly polarized according to the magnetic lines. Model $\mathrm{B}$ gives qualitatively the same results. We see that arrival directions do not cluster around any particular point.

We may conclude that in order to observe one high energy event, for instance, in the direction of the Fly's Eye event we should have observed about 200 in the directions of the galactic magnetic field with similar (or higher) energy. This is just a rough estimation due to the approximations involved in the box chosen and since we don't take into account the exposure of the detectors to the different regions of the galactic plane but it is a significative one. Alternatively, if we normalize the calculated angle integrated flux to the observed flux, we see that the flux in the arrival direction of the observed events must be $\sim 100$ lower than observed and no event could have been seen.

\section{CONCLUSIONS}

In conclusion, magnetic monopoles are very unlikely the cosmic ray particles at the highest energies. Expected fluxes from monopoles are highly anisotropic, pointing towards the magnetic lines near the Earth. Moreover, the energy of the monopoles should be much higher than observed and the spectrum, basically flat, contrary to observation. These results are based only in the structure of the magnetic field. A random component of the magnetic field could change this picture only if it is larger than the regular component.

\section{Acknoledgements}

The authors thank M.C. Gonzalez-Garcia for discussions on the subject. RAV thanks the IFT for its kind hospitality and for providing us with computer resources. RAV's work 
was supported by FAPESP. 


\section{REFERENCES}

[1] T.J. Weiler and T.K. Kephart, Nucl.Phys.Proc.Suppl 51 B (1996) 218. preprint astroph/9605156,

[2] T.W. Kephart and T.K. Weiler, Astroparticle Phys. 4 (1996) 217.

[3] E.N. Parker, Astrophys. Jour. 160 (1970) 383.

[4] E.M. Purcell, in Magnetic Monopoles Ed. R.A. Carrigan and W.P. Trower, Plenum Press.

[5] T.W.B. Kibble, J. Phys. A9 (1976) 1387, Phys. Rept. 67 (1980) 183.

[6] T. Stanev, preprint, astro-ph/9607086.

[7] J.P. Vallée, Astrophys. Jour. 366 (1991) 450.

[8] N. Hayashida et al. Phys. Rev. Lett. 77 (1996) 1000.

[9] T. Stanev, P.L. Biermann, J. Lloyd-Evans, J. Rachen and A.A. Watson, Phys. Rev. Lett. 75 (1995) 3056. 


\section{FIGURES}

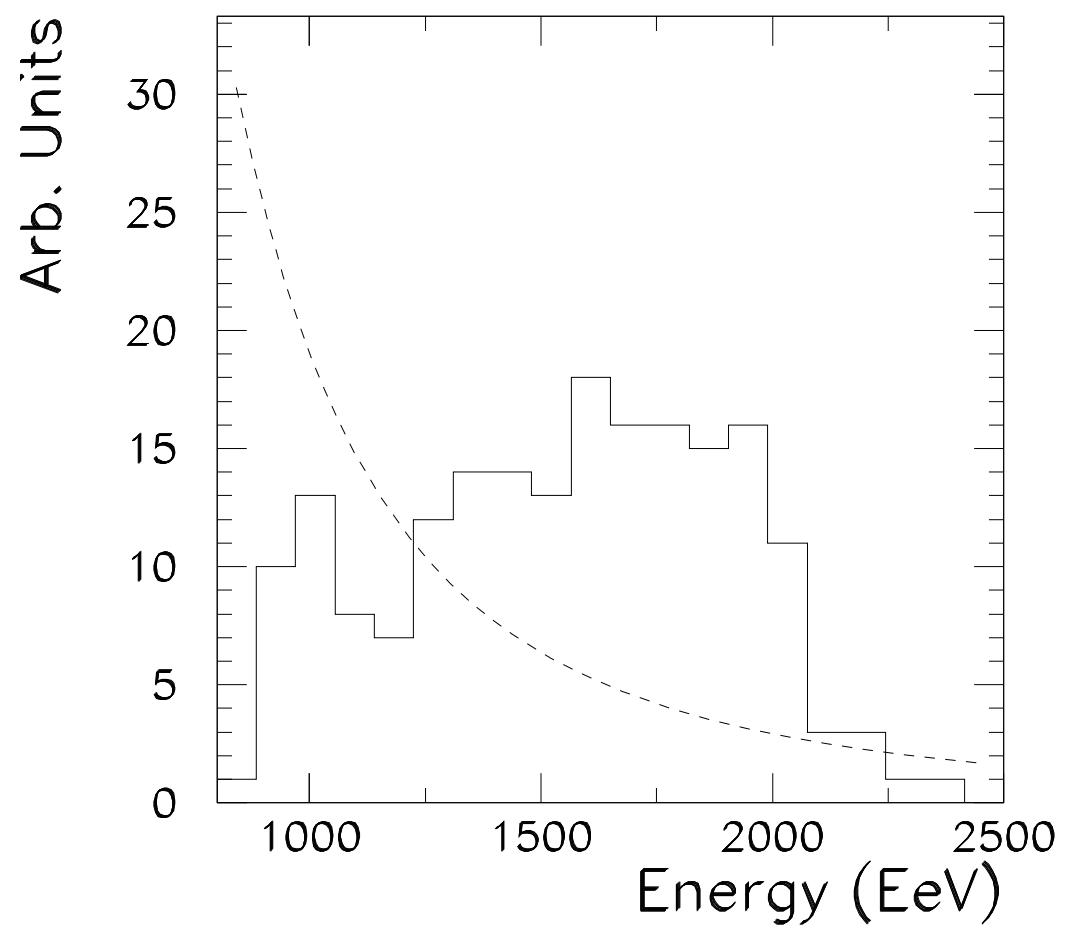

FIG. 1. Energy spectrum of monopoles coming from the center of the Galaxy for the Model A of the magnetic field. Dashed line shows a typical power law HECR spectrum $\sim E^{-2.7}$, normalized to the same number of events. 


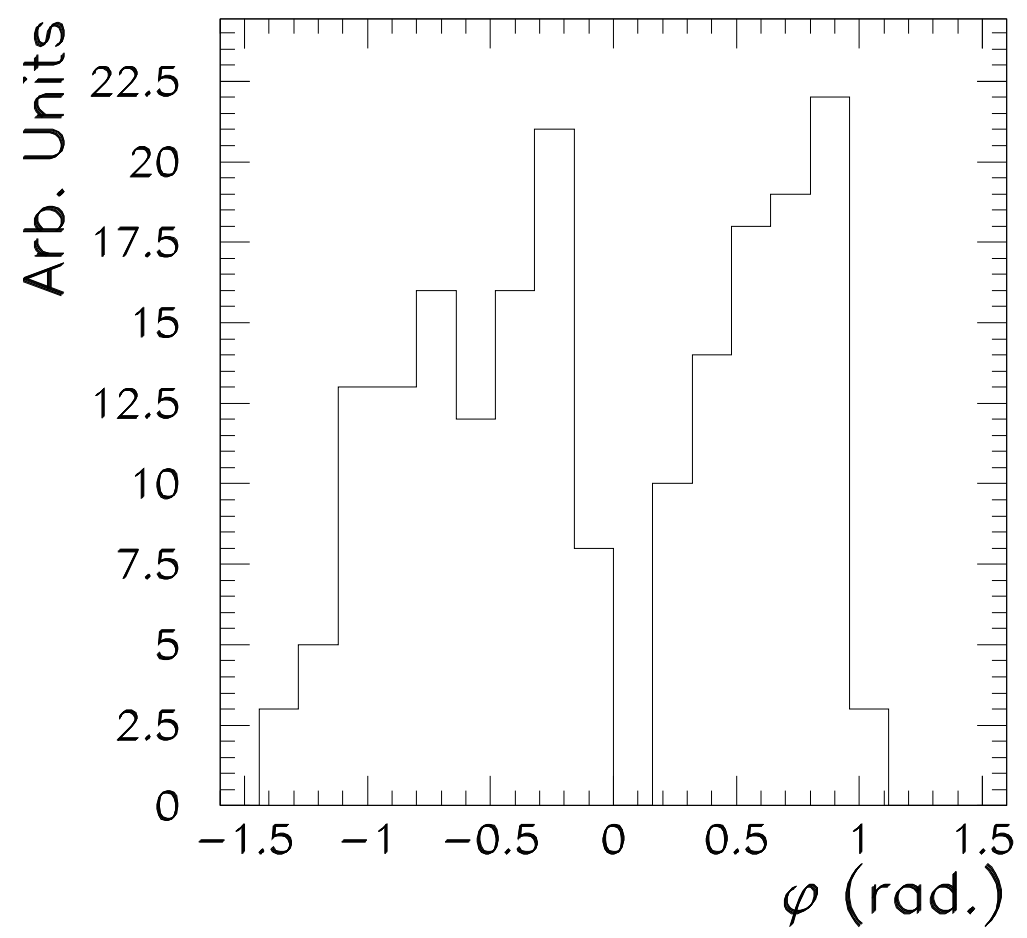

FIG. 2. Arrival direction (in galactic longitude, radians) for monopoles coming from the center of the galaxy. 

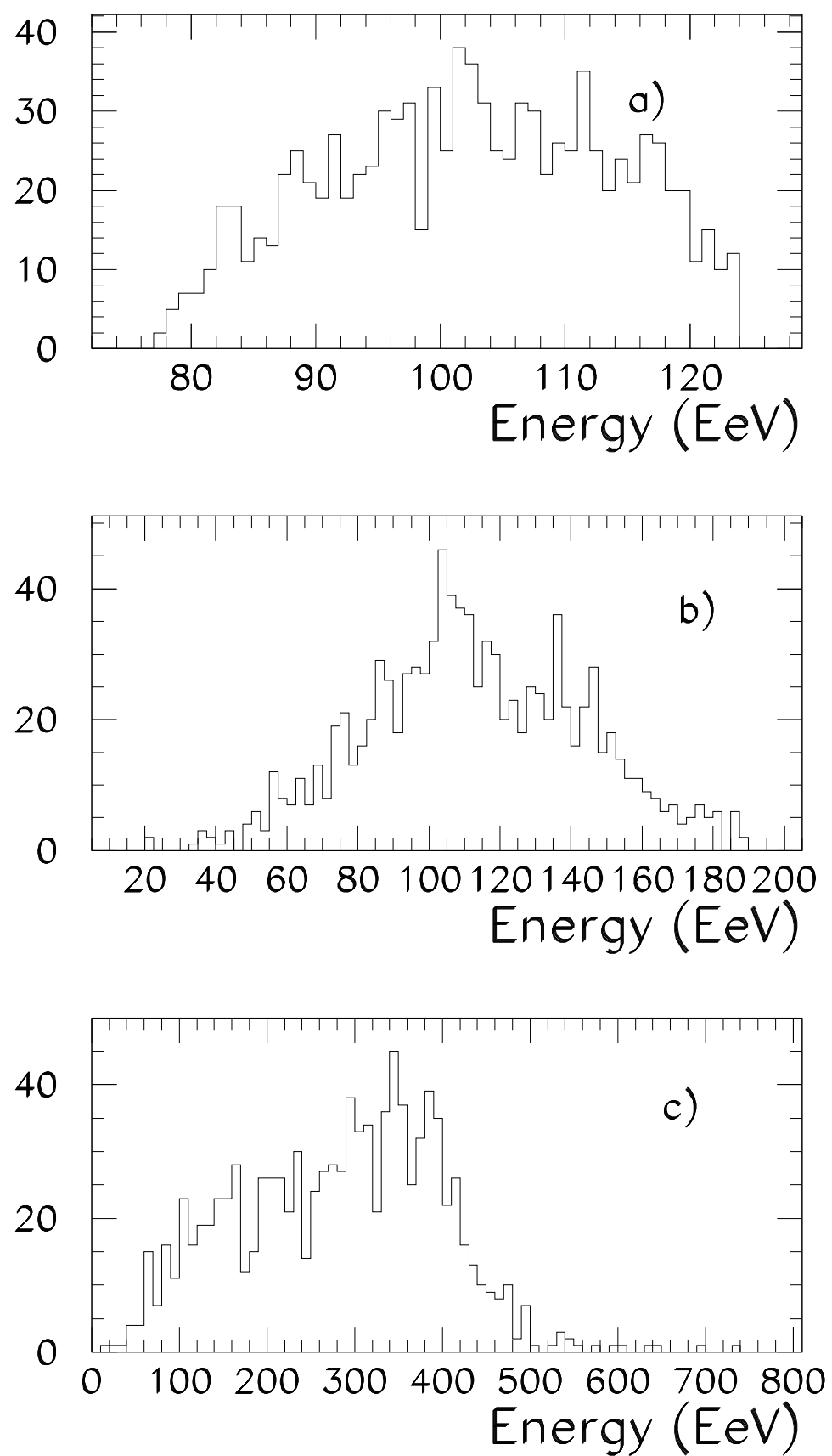

FIG. 3. Energy spectrum of monopoles reaching the Earth with fix energy, $E=100 \mathrm{EeV}$, after they are backpropagated a) 300 pc, b) 900 pc, c) $3 \mathrm{kpc}$. 

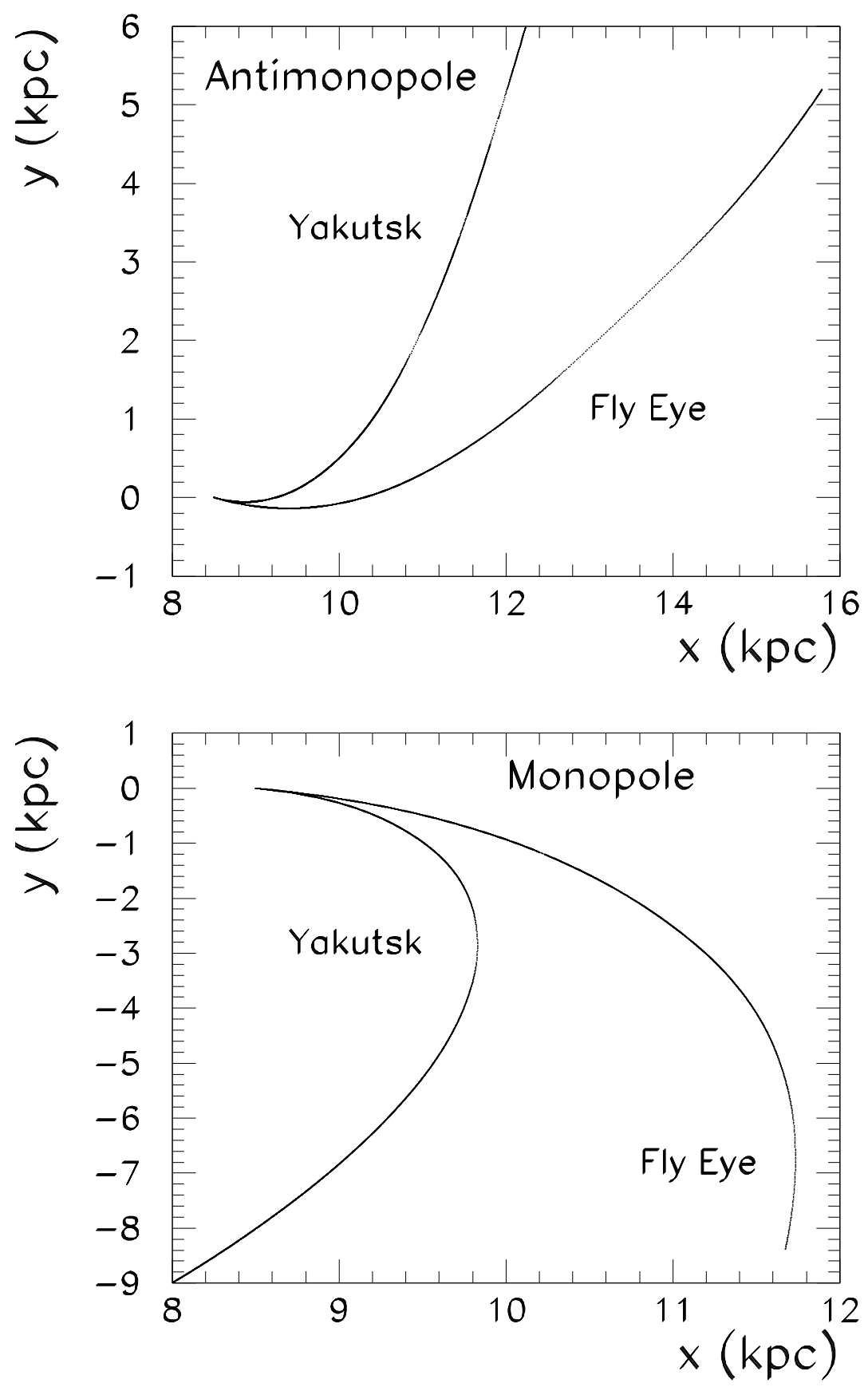

FIG. 4. Trajectory on the $x y$ plane (in kpc) of the highest energy events detected by Fly's Eye and by Yakutsk, assuming they are antimonopoles (upper figure) or monopoles (lower figure). 

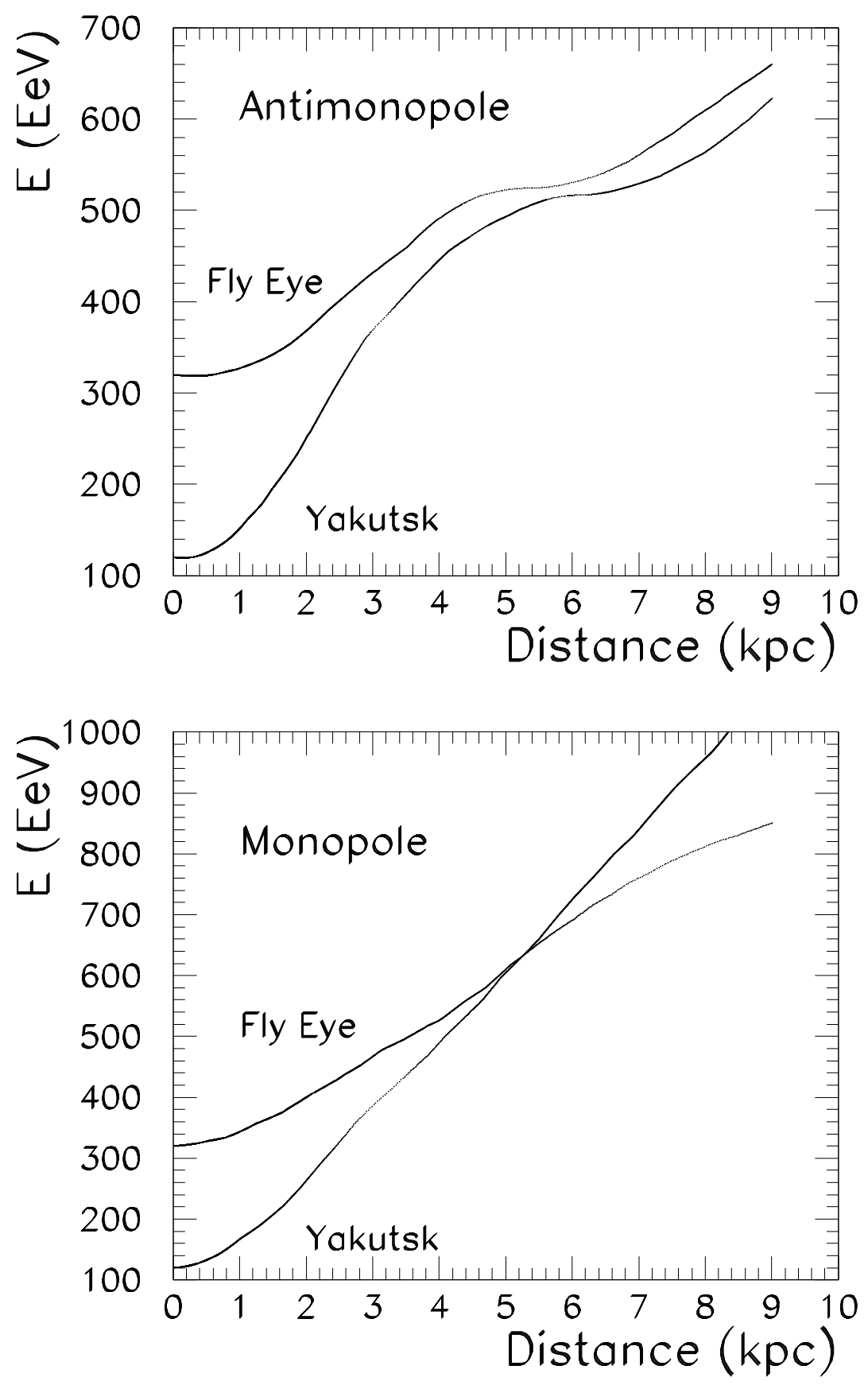

FIG. 5. Monopole energy as a function of the distance to the Earth for the two events of the previous figure. 


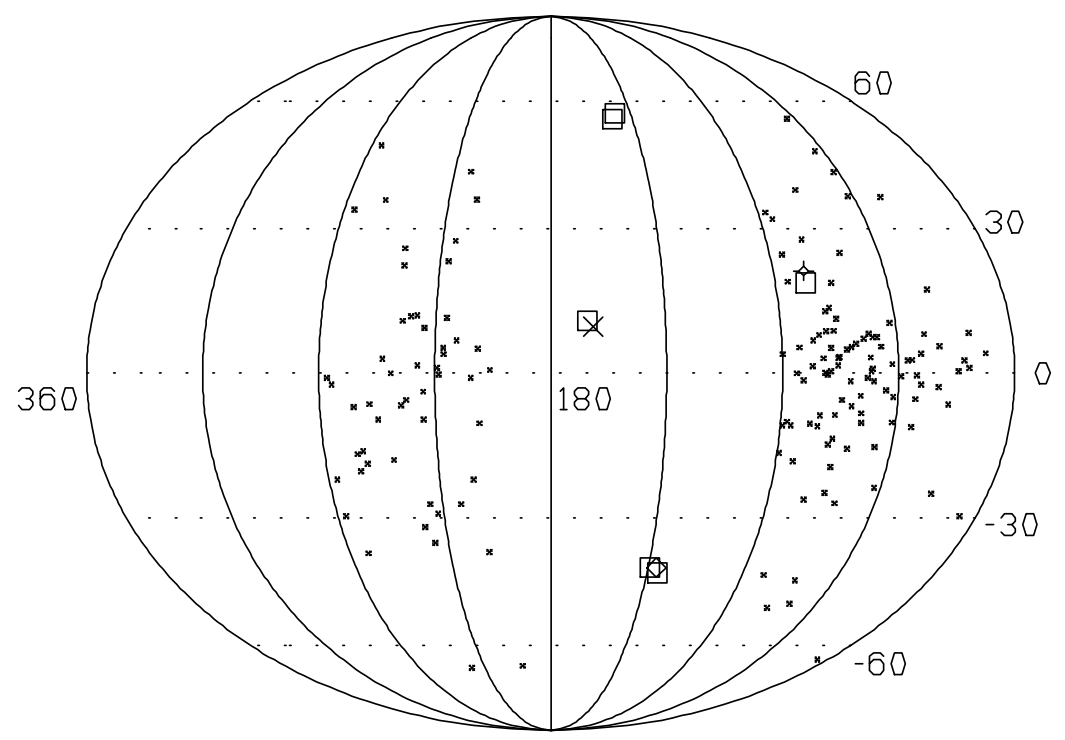

FIG. 6. Arrival direction in galactic coordinates of monopoles seed uniformly in the galaxy and arriving near the Earth (small dots). High energy observed events are represented by squares (Akeno events), crosses (Fly's Eye) and diamonds (Haverah Park). 